\title{
La profesionalización de la secretaria y el servicio al cliente en la ciudad de Portoviejo
}

\section{The professionalization of the secretary and customer service in the city of Portoviejo}

La profesionalización de la secretaria

Silvia Pico Bazurto ${ }^{(1)}$

Lorena Adrián Loor ${ }^{(2)}$

Sonia Úbillus Saltos ${ }^{(3)}$

Daniela Toromoreno Romero ${ }^{(4)}$

(1) Instituto Superior Tecnológico Portoviejo, Manabí. Ecuador. email: picosilvia@ hotmail.com.ar ORCID: https://orcid.org/0000-0003-0649-2854

(2) Instituto Superior Tecnológico Portoviejo, Manabí. Ecuador. email: ladrian68@ hotmail.com ORCID: https://orcid.org/0000-0001-9906-6357

(3) Instituto Superior Tecnológico Portoviejo, Manabí. Ecuador. email: soniaubi@live.com

(4) Instituto Superior Tecnológico Portoviejo, Manabí. Ecuador. email: angidani5@ hotmail.com Contacto: picosilvia@hotmail.com.ar

Recibido: 18-02-2020

\section{Resumen}

La investigación surgió por la necesidad de conocer el nivel de satisfacción de los usuarios ante la atención de las secretarias en las empresas públicas de la ciudad de Portoviejo, teniendo como diagnóstico inicial que existen deficiencias en la calidad de atención y sobre todo en la capacidad de solución ante los requerimientos de los usuarios. El objetivo del estudio fue identificar el nivel de satisfacción de los usuarios que reciben atención de las secretarias en las empresas públicas de la ciudad de Portoviejo en Ecuador. El estudio es de tipo descriptivo, longitudinal, se lo realizó durante el año 2019, se aplicaron métodos teóricos como el análisis-síntesis, inductivo-deductivo e histórico-lógico; 
como métodos empíricos se usó la encuesta dirigida a usuarios y secretarias de las empresas públicas; como resultado global de la investigación se pudo identificar que la población muestra bastante apatía y desinterés o despreocupación por la calidad de atención que reciben, se pudiera considerar que existe cierta resignación al ver que han puesto quejas anteriores por las deficiencias en la atención y hasta maltrato, sin embargo, las autoridades de dichas empresa poco han hecho por remediar el asunto, concluyendo de manera general que existe la necesidad de mejorar la calidad de atención al público por parte de las secretarias que laboran en las empresas públicas de la ciudad de Portoviejo.

Palabras clave: calidad de atención, usuarios, administración pública, empresas públicas.

\section{Summary}

The investigation arose due to the need to know the level of user satisfaction with the attention of the secretaries in the public companies of the city of Portoviejo, having as an initial diagnosis that there are deficiencies in the quality of care and especially in the ability to solution to user requirements. The objective was to identify the level of satisfaction of the users who receive attention from the secretaries in the public companies of the city of Portoviejo in Ecuador. The study is descriptive, longitudinal, it was carried out during the year 2019, theoretical methods were applied such as analysis-synthesis, inductivedeductive and historical-logical; As empirical methods, the survey aimed at users and secretaries of public companies was used; As a global result of the investigation, it was possible to identify that the population shows enough apathy and disinterest or lack of concern for the quality of care they receive, it could be considered that there is some resignation when they see that they have made previous complaints about the deficiencies in care and even abuse However, the authorities of these companies have done little to remedy the matter, concluding in general that there is a need to improve the quality of customer service by the secretaries working in public companies in the city of Portoviejo.

Keywords: quality of care, users, public administration, public companies

\section{Introducción}

En la actualidad la eficiencia de un secretario/a es esencial en cuanto a la organización de una empresa, ya sea esta de negocios o una industria, el trabajo discreto y proactivo contribuye al funcionamiento de estas, la calidad del servicio que desempeñan es crucial para la imagen empresarial que la empresa o 
institución enfoque hacia sus usuarios, siendo estos participes activos de un trato al cliente con calidad de servicio.

La labor que desempeñan está enfocado a la asistencia ejecutiva de tal manera que son encargados de la recepción y envió de documentación, organización y gestión de archivos y de la recepción de llamadas y agendamiento de turno o visitas en la agenda del jefe. Pero en los últimos tiempos el concepto de secretaria ha cambiado notablemente: ahora ha pasado a ser la asistente perfecta de su jefe, hasta el punto de ser capaz incluso de asumir responsabilidades de éste con credibilidad. (Bozada \& Cañarte, 2017)

En este contexto observamos que actualmente su trabajo es arduo y de responsabilidad absoluta al ser estos/as quienes se encargaran de la gestión de documentación clave dentro de la empresa y de esta manera propiciar una labor más directa y comprometida, facilitando notablemente el trabajo de su jefe.

Ahora es tomada de otra concepción en relación a décadas atrás en que la secretaria era simple y llanamente una persona que sin el más mínimo conocimiento podía sentarse y manipular una máquina de escribir, tener un rostro agradable y buena presencia y ser solo receptiva de órdenes, no obstante en aquellas épocas las organizaciones no tenían mayor desempeño debido a que las exigencias del mercado laboral no eran tan demandantes y cuando la tecnología empezó a reemplazar sus funciones, estos se vieron amenazados, aunque fue solo un rumor que años después se ha vuelto una falacia.

Dentro del desempeño en el área de las secretarias es de suma importancia además de aquel rol administrativo que desempeñan, el trato al cliente y la atención que desde su dependencia les propician, sabiendo que existen muchos factores que determinan un buen trato y que ello es motivo principal de que los secretarios hoy en día se preparen y alcancen una profesionalización que les permita profundizar los conocimientos en pos de contribuir favorablemente al desarrollo empresarial.

El servicio al cliente tiene tres pilares fundamentales, la eficacia, la oportunidad, y la atención. La eficacia a la luz del cliente, significa que se hagan las cosas como el espera (para la empresa significa que se hagan las cosas como lo ha prometido al cliente); esa es la esencia del servicio al cliente y lo que este espera fundamentalmente. Pero no basta con que las cosas se hagan como el cliente espera o como se le ha prometido, es necesario que estas se hagan dentro de unos periodos de tiempo convenidos o racionalmente esperados, y especialmente que se atienda al cliente de una manera amable, respetuosa, cálida y sobre todo humana (Hoyos, 2009). 
Es necesario que la atención al cliente sea de la más alta calidad, información veraz, concreta y precisa, con un nivel de atención adecuado para que los clientes que recibe la información, no solo tenga la idea de un producto, sino además de la calidad del capital humano y técnico con el que va a establecer una relación comercial (Najul-Godoy, 2011).

Es por ello que actualmente es sumamente necesario que los secretarios ejecutivos, brazo derecho de los CEO’S y directivos de las empresas, tengan conocimiento profesionales del área en el que se desenvuelven, y reciban constante capacitación, de ello dependerá que su intervención a la hora del manejo de clientes sea el propicio para la realización de un negocio o de crear un impacto de una buena imagen corporativa, capaz de crear lazos interinstitucionales y el bueno nombre de la empresa en la mente del posible consumidor

Con el paso de los años, estas profesionales han cobrado cada vez más protagonismo en los asuntos corporativos. Y es que desde hace tiempo dejaron de tener entre sus tareas primordiales redactar documentos, contestar llamadas telefónicas y enviar faxes. Hoy manejan varios idiomas y están altamente preparadas para crear, innovar e implementar iniciativas; preparar presentaciones de productos o servicios; concertar reuniones; realizar eventos, y atender a proveedores y clientes. (Clavería, 2010)

Para ello el capital humano que está en contacto directo con el cliente debe tener la formación y capacitación adecuada para tomar decisiones y satisfacer las necesidades de los clientes. Najul-Godoy, (2011), plantea que es por ello que la formación profesional de las secretarias es fundamental para que presten un servicio de calidad y de esa manera llegar a la satisfacción del usuario.

La satisfacción del cliente es vivida hoy en el mundo empresarial como un nuevo credo que concita entusiasmos y afecciones sinceras. Larrea (1991), asevera que el cliente es la principal estrategia de mercadeo de una institución, es el primer portavoz de la calidad del servicio que se otorga dentro de la empresa y se lleva la imagen otorgada, es importante brindar una atención eficaz para que la publicidad y promoción de la institución sea pertinente al enfoque de la misión y visión respectiva.

El capital humano $(\mathrm{CH})$ es el principal componente de cualquier organización contemporánea, pues es el elemento que une, articula y potencia los demás capitales que conforman su capital intelectual, y es la base de su estructura para poder competir exitosamente en la actual era de información y conocimiento. Por otra parte el personal de una institución es clave en el éxito empresarial, siendo que los secretarios 
son la imagen de una empresa y el primer contacto con el público, deben articular todos los valores y principios que la empresa quiere que sus clientes perciban, desde la confianza hasta el servicio pertinente y eficaz (Vázques \& Gabalán, 2011).

\section{Materiales y métodos}

El estudio es de tipo descriptivo, longitudinal, se lo realizó durante el año 2019, se aplicaron métodos teóricos como el análisis-síntesis, inductivo-deductivo e histórico-lógico; como métodos empíricos se usó la encuesta dirigida a usuarios y secretarias de las empresas públicas, la variable de estudio fue la “eficacia del servicio" en las secretarias que atienden al público.

Se trabajó con una muestra de 200 usuarios de empresas públicas de la ciudad de Portoviejo, en Ecuador, que fueron atendidos por secretarias. El nivel de significancia de la investigación fue de +- 5\%, el criterio de inclusión fue secretarias de empresas públicas de la ciudad que trabajen en áreas de atención al público, se excluyeron a secretarias que comparten su labor entre atención al público y trabajo administrativo con sus jefes.

\section{Resultados}

Los resultados que se presentan a continuación representa la información obtenida de la encuesta aplicada a los usuarios de las empresas públicas de la ciudad de Portoviejo, que fueron atendidos en sus requerimientos por personal de secretaria, el objetivo de la encuesta fue identificar la eficiencia en la atención por parte de las secretarias y la satisfacción por la calidad del servicio. Los resultados obtenidos se presentan en la tabla 1 .

En cuanto al nivel de satisfacción con respecto a la agilidad y rapidez de su trámite o servicio, en la información presentada en la tabla 1, se observa que los usuarios tiene un nivel de respuesta a la satisfacción no clara, y más bien un poco confusa, ya que están acostumbrados al nivel de servicio poco satisfactorio que reciben en el sector público, siendo esa la diferencia del sector privado.

\begin{tabular}{|l|l|l|}
\hline Alternativas & Frecuencia & Porcentaje \\
\hline Muy satisfecho & 45 & $22.5 \%$ \\
\hline Satisfecho & 65 & $32.5 \%$ \\
\hline Algo satisfecho & 34 & $17.0 \%$ \\
\hline Insatisfecho & 41 & $20.5 \%$ \\
\hline Muy insatisfecho & 15 & $07.5 \%$ \\
\hline
\end{tabular}




\begin{tabular}{|l|l|l|}
\hline Total & 200 & $100 \%$ \\
\hline
\end{tabular}

Tabla 1. Agilidad y rapidez en el trámite o servicio

Al momento de la aplicación del instrumento se pudo apreciar además que los usuarios poco utilizaban el dispositivo electrónico implementado en las empresas públicas para evaluar la gestión de las secretarias. Hubo usuarios que no evaluaban, demostrando poco interés a la mejora del servicio y otros lo hacían con tanta rapidez, sin reflexionar en la importancia que tienen estos resultados para superar la calidad de atención al público, presentándose los resultados en la tabla 2.

\begin{tabular}{|l|l|l|}
\hline Opciones & Frecuencia & Porcentaje \\
\hline Muy satisfecho & 32 & $16.0 \%$ \\
\hline Satisfecho & 55 & $27.5 \%$ \\
\hline Algo satisfecho & 38 & $19.0 \%$ \\
\hline Poco satisfecho & 40 & $20.0 \%$ \\
\hline Nada satisfecho & 35 & $17.5 \%$ \\
\hline Total & 200 & $100 \%$ \\
\hline
\end{tabular}

Tabla 2. Desempeño en el servicio solicitado (ejecución de manera correcta y a la primera vez)

El nivel de satisfacción en cuanto al desempeño del servicio solicitado se muestra en la tabla 2, en la cual se puede evidenciar un alto porcentaje de "poco satisfechos" y "nada satisfechos" observando de esta manera que la eficacia del servicio en cuanto a la calidad de atención de la secretaria es deficiente, considerando que lo ideal es que toda la población quede muy satisfecha o al menos satisfecha por el servicio o trato recibido, sobre este tema se puede acotar también que no se pudo observar iniciativas de cambio por parte de las secretarias, tabla 3.

\begin{tabular}{|l|l|l|}
\hline Opciones & Frecuencia & Porcentaje \\
\hline Muy fácil & 57 & $28.5 \%$ \\
\hline Regularmente fácil & 38 & $19.0 \%$ \\
\hline Poco fácil & 88 & $44.0 \%$ \\
\hline Nada fácil & 17 & $8.50 \%$ \\
\hline Total & 200 & $100 \%$ \\
\hline
\end{tabular}

Tabla 3. Percepción del procedimiento que llevo a cabo para realizar su trámite o servicios solicitados

El usuario percibe la organización y comunicación en el área o departamento para la realización de su trámite de forma heterogénea, por un lado lo considera bueno un 44\%, y por el otro lado un $27 \%$ lo considera pésimo, así se muestran los resultados en la tabla 4. Este nivel de percepción de insatisfacción lleva a concluir que pese a que la calidad del servicio es efectivo, aun no alcanza los estándares de atención que satisfagan las necesidades de los clientes, los resultados se presentan en la tabla 4. 


\begin{tabular}{|l|l|l|}
\hline Opciones & Frecuencia & Porcentaje \\
\hline Excelente & 32 & $16.0 \%$ \\
\hline Bueno & 88 & $44.0 \%$ \\
\hline Regular & 26 & $13.0 \%$ \\
\hline Pésimo & 54 & $27.0 \%$ \\
\hline Total & 200 & $100 \%$ \\
\hline
\end{tabular}

Tabla 4. Percepción de la organización y comunicación en el área o departamento para la realización del trámite o servicio solicitado

\section{Discusión}

Las variables de investigación que se presentan en este documento fueron: agilidad en trámite, desempeño de las secretarias y organización en la información. Se tomó aleatoriamente a los usuarios luego de que fueron atendidos por las secretarias en las empresas públicas para responder a los requerimientos planteados por el equipo de investigadores, en particular, no demandó más de tres minutos, por el riesgo que conlleva quitarles mucho tiempo ya que habían esperado para ser atendidos, el nivel de aceptación a la encuesta fue alta.

Esta investigación, desde su planificación, implementación y publicación de resultados, ha ayudado de una manera significativa al mejoramiento de la calidad de las secretarias en sentido general, enfatizando en la comunicación entre las distintas áreas de la empresa y el control sobre la calidad de atención al cliente, lo que contribuye a su pertinencia y aporte social.

Se concuerda con otros autores que refieren que la secretaria es la persona que escribe la correspondencia, resuelve los asuntos de trámite y custodia los documentos de una oficina, es decir es quien asegura el eficaz funcionamiento de la empresa (Clavería; 2015; Bozada \& Cañarte 2017).

El instrumento aplicado tuvo su prueba piloto, técnica que permitió mejorarlo y validar su aplicación durante los meses de noviembre y diciembre del año 2019, meses de temporada alta por así decirlo, en cuanto a trámites en las empresas públicas. Tal como se aspiraba, la investigación planteada incorporó como estrategia la devolución ética de los resultados a los gerentes de las empresas para que conocieron los resultados de la investigación, con esto se logró que: a) dejaran de existir trámites inconclusos, evitando errores y confusiones por parte de las secretarias; b) los clientes conocieran el detalle de los trámites relacionados a sus necesidades o requerimientos; c) las secretarias se limitaran realizar actividades estrictamente relacionadas con su puesto de trabajo y faciliten los requerimientos de los 
usuarios; y, d) las secretarias conocieran anticipadamente la disponibilidad de insumos para su trabajo y que no se paralice la atención al usuario por una eventual falta de insumos o recursos tecnológicos.

La observación realizada durante el proceso de aplicación de la encuesta, permitió identificar la incorporación del software que complementa o facilita el servicio al usuario. En las salas de espera existen equipos de distracción para soportar la espera, lo que ayuda a que supuestamente, disminuyeran los tiempos de espera en la atención de los clientes hasta ser escuchados en sus pedidos, y se pudieran anticipar actividades vinculadas al procedimiento que se lleva en esa dependencia. Por otra parte, la apariencia de las secretarias no en todos los casos brindó confianza como para que los usuarios se sintieran alentados a manifestar sus requerimientos de forma oportuna y disminuir significativamente el tiempo en su atención. Como consecuencia de los cambios impulsados, los tiempos medios de espera en los meses de alta demanda, se espera disminuyan de 15 a 30 minutos, y los tiempos medios de desfase entre la atención de uno a otro cliente descienda también en el tiempo.

Se concuerda con Cedeño y Vera (2015), en la que señalan que las secretarias deben tener competencias y habilidades en ciertas materias que le permitirán desempeñar sus funciones con la debida eficacia profesional: mecanografía, ortografía y gramática, caligrafía, matemática financiera, contabilidad, idioma extranjero y ofimática. Es evidente que al contar con una buena preparación les va a permitir una mejor atención a los clientes.

\section{Conclusiones}

La calidad de atención al cliente se puede medir desde diferentes indicadores. Desde el punto de vista de la agilidad y rapidez en el trámite que realizaron los usuarios atendidos por secretarias de las empresas públicas de la ciudad de Portoviejo, no se logró alcanzar un nivel de satisfacción que garantice excelencia, la época en que se vive, alienada por tecnología en todos los procesos, ha simplificado el trabajo administrativo, ante estas perspectivas, resulta sumamente importante que se priorice el tema del tiempo y la agilidad en los servicios ofrecidos.

El desempeño de las secretarias se lo puede resumir como aceptable, la información obtenida da cuenta de que no se ha logrado un nivel de excelencia total en todas las secretarias que atienden al público, esto se debe a la heterogeneidad en la formación que tienen las personas que cumplen las funciones de 
secretarias, muchas de ellas no cuentan con formación secretarial, sino que por circunstancias de la vida y por necesidades laborales cubren las plazas de secretarias sin estar formadas como tales.

Los usuarios atendidos por las secretarias de las empresas públicas, poca importancia le dan a los procesos de evaluación del desempeño y la calidad del servicio recibido. Muchos de los usuarios se muestran indiferentes a evaluar el desempeño de las secretarias y los pocos que lo hacen, lo efectúan de manera superficial, rápida y solo por cumplir con uno de los pasos del proceso. Esto da a pensar que los resultados de la información que ofrecen en los dispositivos electrónicos que evalúan la gestión no surten efectos en las decisiones que tomen los jefes relacionadas con mejorar la calidad de atención al cliente.

\section{Bibliografía}

Bozada, S., \& Cañarte, T. (2017). Algunas consideraciones sobre el desempeño de la secretaria ejecutiva. La nueva tecnología, herramienta fundamental. Revista Científica Dominio de las Ciencias, 92.

Cedeño, D. \& Vera, M. (2015). Archivo tradicional y computarizado en las funciones administrativas de las secretarias de las instituciones públicas y privadas, financieras. Recuperado dehttp://repositorio.utm.edu.ec/bitstream/123456789/105/1/EL\%20ARCHIVO\%20TRADICIONA L\%20Y\%20COMPUTARIZADO\%20EN\%20LAS\%20FUNCIONES\%20ADMINISTRATIVAS \%20DE\%20LAS\%20SECRETARIAS\%20DE\%20LAS.pdf

Clavería, A. (2010). El nuevo perfil de las secretarias ejecutivas. Recuperado de https://mba.americaeconomia.com/articulos/reportajes/el-nuevo-perfil-de-las-secretarias-ejecutivas

Hoyos, R. (2009). La auditora del servicio al cliente a través de la observación participante: "El cliente incognito". Revista Colombiana de Marketing , 36.

Larrea, P. (1991). Calidad de Servicio del Marketing a la estrategia . Madrid España: Ediciones Díaz de Santos .

Najul-Godoy, J. (2011). El capital humano en la atención al cliente y la calidad del servicio . Observatorio Laboral , 26.

Vázques, F., \& Gabalán, J. (2011). Implementación de un modelo de administración de capital humano en un grupo de investigación . El profesional de la información , 517. 